\title{
Une pluralité de lieux au cœur de l'innovation
}

Federica Minichiello

\section{(2) OpenEdition}

Journals

Édition électronique

URL : https://journals.openedition.org/ries/8870

DOI : $10.4000 /$ ries.8870

ISSN : 2261-4265

Éditeur

France Education international

Édition imprimée

Date de publication : 1 décembre 2019

Pagination : 12-15

ISBN : 9782854206258

ISSN : 1254-4590

Référence électronique

Federica Minichiello, «Une pluralité de lieux au cœur de l'innovation », Revue internationale d'éducation de Sèvres [En ligne], 82 I décembre 2019, mis en ligne le 01 décembre 2019, consulté le 15 janvier 2022. URL : http://journals.openedition.org/ries/8870 ; DOI : https://doi.org/10.4000/ries.8870

Ce document a été généré automatiquement le 15 janvier 2022.

(c) Tous droits réservés 


\title{
Une pluralité de lieux au cœur de l'innovation
}

\author{
Federica Minichiello
}

1 Tiers-lieux, incubateurs et accélérateurs, fab-labs, espaces de travail collaboratif : la profusion de lieux d'innovation, en France et dans le monde, reflète l'encouragement d'un apprentissage informel et coopératif, d'une culture de la création qui trouve son expression dans des espaces dits « de connaissance et de culture».

2 Un guide sur le campus d'avenir ${ }^{2}$, publié en 2015 par la Direction générale de l'enseignement supérieur et de l'insertion professionnelle, illustre la traduction dans l'espace d'une pédagogie collaborative (souvent numérique) avec l'émergence, à côté de la salle de classe, de nouveaux lieux, "connectés et confortables", définis comme « espaces capables »: des espaces polyvalents, capables de répondre simultanément à une grande variété d'usages grâce à une offre de services et d'équipement indissociable du lieu physique.

3 Le présent article se concentre initialement sur le processus d'innovation, principale identité et raison d'être de ces lieux souvent multiformes et propose quelques ressources sur les modalités de mise en œuvre dans le domaine de l'éducation. Sitographie arrêtée le $1^{\text {er }}$ décembre 2019.

\section{Le processus d'innovation}

\section{Comprendre l'innovation}

4 La banque publique d'investissement, Bpifrance, est une filiale de la Caisse des dépôts, créée en 2012. Son pôle «Bpifrance hub » a pour mission d'accompagner les start-up et les petites et moyennes entreprises dans leur processus de transformation. L'innovation est définie comme un processus centré, dès son origine, sur les utilisateurs, sur leurs besoins et sur leur expérience, qui tend à la conception d'un produit ou d'un service nouveau (ou significativement amélioré) ; l'innovation est, pour de nombreux acteurs, un facteur de différentiation et presque une nécessité 
stratégique. Après une décennie d'innovation impulsée par la transformation digitale et la généralisation des appareils mobiles et connectés, l'actualité des investissements est dans les technologies dites « de rupture ", ou deep-tech, des technologies qui requièrent des recherches complexes et des années de maturation mais qui auront un impact significatif sur les habitudes des utilisateurs.

https://lehub.web.bpifrance.fr

\section{Mesurer l'innovation}

5 Le tableau de bord européen de l'innovation (TBEI) est une initiative de la direction générale du marché intérieur, de l'industrie, de l'entrepreneuriat et des PME de la Commission européenne. Il s'agit d'une évaluation annuelle de la performance des États membres en termes de recherche et d'innovation, accompagnée d'une analyse des leviers d'amélioration. Trois dimensions clés sont à la base de l'innovation : le capital humain, la recherche et l'écosystème. Parmi les variables de mesure utilisées on peut citer le niveau d'investissement public et privé, le taux de pénétration de la connexion à haut débit, le nombre de co-publications scientifiques internationales, les dépenses publiques en recherche et développement, etc. Au palmarès 2019 des pays les plus innovants : la Corée du Sud, le Canada, l'Australie, le Japon et, en Europe, le Danemark, la Finlande, les Pays-Bas et la Suède.

https://interactivetool.eu/EIS/index.html

\section{S'inspirer de bonnes pratiques}

6 L'Observatoire de l'innovation dans le secteur public (OPSI) est une initiative de l'OCDE pour promouvoir l'innovation dans le secteur public, avec le soutien financier de la Commission européenne dans le cadre du programme Horizon 2020. L'OPSI aide et conseille les administrations publiques en leur mettant à disposition des outils comme le «Toolkit Navigator », une base de connaissance recensant plus d'une centaine d'outils d'innovation et de méthodologies novatrices de transformation du secteur public, accompagnées d'études de cas.

https://oecd-opsi.org/

\section{Typologies de lieux}

\section{Le learning center}

7 Le learning center est une forme de lieu d'apprentissage : c'est un espace de vie, ouvert, facilitant l'échange et la collaboration et qui propose une diversité de modalités d'accès et de services. Traditionnellement considéré comme l'évolution d'une bibliothèque, où les ressources ne constituent plus le (seul) cœur de l'offre, il présuppose un changement organisationnel, avec un personnel qui devient médiateur du savoir et un management par la valeur, en réponse aux besoins des usagers. Le ministère de l'enseignement supérieur et de la recherche a publié en 2012 un guide d'aide à la conception d'un learning center, dans le cadre de la construction ou de la réhabilitation d'une structure.

https://bit.ly/33FbL3L 


\section{Fab-lab}

Un laboratoire de fabrication digitale ( Fab-lab») est un lieu qui donne accès à un environnement, des compétences, des matériaux et des technologies propices à la création et à l'invention. La Fab Foundation, organisation américaine à but non lucratif issue d'un programme du Massachusetts Institute of Technology (MIT), promeut le développement d'un réseau international de ces laboratoires, notamment au sein des établissements d'enseignement. Le réseau Fablabs.io [https://www.fablabs.io/] compte plus de 1750 lieux autour du monde, dans une centaine de pays.

https://fabfoundation.org

\section{Tiers-lieu}

9 Le terme «tiers-lieu » se réfère aux principaux lieux de vie : le domicile (premier lieu) et le lieu de travail (deuxième lieu) : le tiers-lieu est un lieu quotidien comme les deux autres, parfaitement intégré dans l'environnement quotidien mais qui est partagé avec d'autres. Sa mission principale est de rassembler et d'encourager l'interaction entre des groupes. Distinct d'un simple espace convivial ou d'échange, il est un lieu de conception et de documentation des projets qui y sont réalisés.

http://www.tierslieuxedu.org/

\section{État des lieux}

10 Le cluster parisien « Innovation Factory », avec Bpifrance et la chaire NewPIC de la Paris School of Busines ont réalisé une série de plusieurs études pour comprendre les spécificités des différents lieux d'innovation qui fleurissent en France et dans le monde. Trois traits communs les réunissent: une communauté d'individus qui se retrouve autour de valeurs communes, apportant une variété d'expériences et de compétences. Un lieu physique qui favorise la rencontre et l'émergence de nouvelles pratiques de collaboration; une offre de services sans cesse renouvelée: incubation, travail collaboratif, ateliers de fabrication, conseil, mentorat, etc. Certains lieux seront plus orientés vers des prestations intellectuelles, d'autres plus centrés sur le prototypage et la fabrication. La dernière étude de 2019 compare l'offre de lieux entre la France et l'Asie : il en ressort une certaine homogénéité de pratiques en termes de gestion de projets d'innovation et de management des communautés, avec une utilisation généralisée de méthodes de créativité comme la pensée design.

https://plateformes-innovation.fr

\section{Des ressources en éducation}

\section{Archiclasse}

11 Le dispositif ministériel français Archiclasse réunit des méthodes et des outils pour accompagner la rénovation ou la construction d'un établissement scolaire, afin d'anticiper l'évolution de l'utilisation des espaces, notamment en lien avec les dispositifs numériques. ArchiLab, nouvel outil de l'écosystème Archiclasse, est un kit 
qui aide les acteurs éducatifs et les collectivités à réfléchir ensemble à la conception d'espaces pédagogiques et à les formaliser, grâce à des exemples d'espaces innovants proposés dans la boîte à outils.

https://archiclasse.education.fr/

\section{Future Classroom Lab}

Le réseau European School Network a publié en 2017 des lignes directrices sur l'exploration et l'adaptation des espaces d'apprentissage dans les écoles [https://bit. ly/37Vbm0A] avec huit cas-pays étudiés, montrant une certaine hétérogénéité entre pays : certains sont plus avancés dans la mise en place d'espaces d'apprentissage plus flexibles, d'autres n'ont pas encore inscrit cet axe dans leurs priorités. Parmi les initiatives du réseau on peut citer aussi le Future Classroom Lab (FCL) un lieu physique qui invite à un parcours à travers les compétences dites du XXI ${ }^{e}$ siècle, organisées en six zones d'apprentissage : l'enquête, pour améliorer la pensée critique. La création en équipe, pour une véritable construction du savoir. La présentation, pour travailler la capacité de communication. L'interaction, pour faire participer activement tous les élèves. L'échange, pour apprendre à collaborer. Le développement, un espace d'apprentissage informel et d'autoréflexion.

http://fcl.eun.org/learning-zones

\section{La pensée design à l'école}

13 La pensée design est une démarche de gestion de projet qui permet d'identifier des solutions à une problématique en partant de l'écoute des besoins des personnes concernées. Cette démarche mobilise des compétences souvent sous-exploitées comme le sens de l'intuition, l'intelligence émotionnelle ou l'esprit pratique. Parmi quelques exemples de «bon réflexe : disposer d'un espace dédié (même un simple mur), comme rappel tangible du travail déjà effectué (images, notes) pour permettre le suivi d'un projet ou reconfigurer l'espace, de temps à autre, pour faire émerger de nouvelles idées. L'association SynLab a publié un manuel de la pensée design pour les enseignants, adapté au contexte spécifique de l'éducation après l'avoir testé sur différentes écoles en France.

https://syn-lab.fr/

\section{Nouveaux espaces d'apprentissage}

14 Le groupe de veille et de prospective OCEAN, piloté par l'Institut français d'éducation (IFé), est l'un des dix groupes initiés par la direction du numérique pour l'éducation du ministère de l'éducation nationale et de la jeunesse en France, sur les objets connectés (robots, capteurs, équipements individuels...) et sur le développement de nouveaux espaces d'apprentissage liés aux technologies numériques (réseaux sociaux, communautés virtuelles, fablabs, etc.). Le dossier de capitalisation des échanges du groupe invite à "élargir et hybrider les espaces et les temps d'apprentissage ", en dépassant les unités de mesure traditionnelles de l'espace et du temps, en ouvrant la classe sur l'extérieur et sur des acteurs « autres » que les enseignants. https://bit.ly/2OFhXEE 


\section{NOTES}

2. https://bit.ly/2OFKAlh

\section{INDEX}

Mots-clés : tiers-lieu, fab lab, learning centre, travail collaboratif innovation, incubation, pensée design, espace d'apprentissage

Palabras claves : tercer lugar, Fab lab, learning centre, trabajo colaborativo, innovación, incubación, pensamiento de diseño, espacio de aprendizaje

Keywords : third places, Fab lab, learning centres, collaborative work, innovation, incubation, design thinking, learning spaces

\section{AUTEUR}

\section{FEDERICA MINICHIELLO}

Federica Minichiello est chargée de mission « Laboratoire numérique de l'éducation » (LNE) et déléguée à la protection des données à France Éducation international. Courriel :

minichiello@ciep.fr 